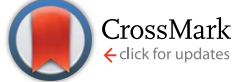

Cite this: RSC Adv., 2017, 7, 17682

Received 10th February 2017 Accepted 17th March 2017

DOI: 10.1039/c7ra01706e

rsc.li/rsc-advances

\section{Effective dopants in p-type elementary Te thermoelectrics $\dagger$}

\author{
Xin Qian, $\$^{a}$ Yu Xiao, $\$^{a}$ Lei Zheng, ${ }^{* a}$ Bingchao Qin, ${ }^{a}$ Yiming Zhou, ${ }^{a}$ Yanling Pei, ${ }^{a}$ \\ Bifei Yuan, ${ }^{b}$ Shangkai Gong ${ }^{a}$ and Li-Dong Zhao*a
}

Elementary $\mathrm{Te}$ is predicted to be a high-performance thermoelectric material through theoretical calculation, and the experimental results have confirmed this prediction through optimizing carrier concentrations. In this work, we investigate the thermoelectric transport properties in undoped Te at 300-673 K, revealing a low thermal conductivity $\sim 1.8 \mathrm{~W} \mathrm{~m}^{-1} \mathrm{~K}^{-1}$ at room temperature and a $Z T \sim 0.2$ at $653 \mathrm{~K}$. To further improve the thermoelectric performance in the Te system, we choose $\mathrm{Sn}, \mathrm{Pb}, \mathrm{Bi}$, and $\mathrm{Sb}$ as dopants in Te. We find $\mathrm{Bi}$ and $\mathrm{Sb}$ can effectively enhance the electrical conductivity by two orders, from $\sim 2 \mathrm{~S} \mathrm{~cm}^{-1}$ in undoped Te to $\sim 300 \mathrm{~S} \mathrm{~cm}^{-1}$ and $\sim 550 \mathrm{~S} \mathrm{~cm}^{-1}$ at $300 \mathrm{~K}$ in Bi-doped and $\mathrm{Sb}$-doped samples respectively. Meanwhile, these dopants can simultaneously significantly suppress the lattice thermal conductivity, decreasing from $1.22 \mathrm{~W} \mathrm{~m}^{-1} \mathrm{~K}^{-1}$ in undoped Te to $0.68 \mathrm{~W} \mathrm{~m}^{-1} \mathrm{~K}^{-1}$ and $0.73 \mathrm{~W} \mathrm{~m}^{-1} \mathrm{~K}^{-1}$ at $673 \mathrm{~K}$ in Bi-doped and Sb-doped samples, respectively. Maximum $Z T$ values of $\sim 0.8$ in $\mathrm{Te}_{0.9925} \mathrm{Bi}_{0.0075}$ and $\sim 1.1$ in $\mathrm{Te}_{0.985} \mathrm{Sb}_{0.015}$ at $673 \mathrm{~K}$ can be achieved. The results indicate that $\mathrm{Sb}$ is an effective dopant in the Te system.

\section{Introduction}

Thermoelectric materials enable direct conversion between waste heat and electric power and provide an alternative for humanity to solve the increasingly severe energy and environment issues. Researchers are making great efforts to explore and design highperformance thermoelectric materials for large-scale practical application. ${ }^{1-8}$ The key parameter that determines the efficiency of thermoelectric materials is the 'dimensionless figure of merit' $Z T$, which is defined as $Z T=\left(\alpha^{2} \sigma / \kappa\right) T$, where $\alpha, \sigma, \kappa$ and $T$ denote the Seebeck coefficient, electrical conductivity, thermal conductivity and working temperature in kelvin, respectively. ${ }^{9-16}$ For a favorable thermoelectric material, it is supposed to exhibit high Seebeck coefficient and electrical conductivity, and minimal thermal conductivity. However, the complex interdependence among these parameters makes it challenging to improve the thermoelectric performance.

Ongoing research efforts initially focus on conventional thermoelectric materials, namely bismuth telluride $\mathrm{e}^{17-19}$ and lead chalcogenides, ${ }^{20-22}$ and also some emerging thermoelectric materials. Among which zintle phase, ${ }^{23}$ skutterudites, ${ }^{24-26}$ copper and tin chalcogenides, ${ }^{27-29}$ oxidation chalcogenide ${ }^{30,31}$ and SnSe crystals $^{32,33}$ appear to be promising thermoelectric materials.

\footnotetext{
${ }^{a}$ School of Materials Science and Engineering, Beihang University, Beijing 100191, China.E-mail: zhenglei@buaa.edu.cn; zhaolidong@buaa.edu.cn

${ }^{b}$ China National Petroleum Corporation Greatwall Drilling Company, Beijing 100101, China

$\dagger$ Electronic supplementary information (ESI) available. See DOI: 10.1039/c7ra01706e

\$ Xin Qian and Yu Xiao contributed equally to this work.
}

Recently, elementary Te is predicated as a potential thermoelectric material due to its particular band structure and inherent band convergence, which can provide additional conducting channels to enhance the electrical conductivity and Seebeck coefficient simultaneously. ${ }^{34-36}$ It is confirmed that Te is characterized by the inherent valence band notification at $\mathrm{H}$ point. Similar situation happened on conduction band as well. This characteristic band structure makes it easy to achieve high power factor via modifying the Fermi level crossing into multi-bands. Experimentally, a peak $Z T$ value $\sim 1.0$ was achieved in polycrystalline Te system by heavy p-type doping. ${ }^{36}$ To further optimize the electrical transport properties of Te, we selected several dopants to verify the most effective dopants in Te system. Additionally, the origins of low thermal transport properties of undoped elementary Te are worth further unclosing.

In this work, we investigated the thermoelectric performance of undoped elementary Te at 300-653 K, prepared using spark plasma sintering (SPS) method. Undoped Te exhibits a low electrical conductivity reaching $\sim 2 \mathrm{~S} \mathrm{~cm}^{-1}$, and a low thermal conductivity $\sim 1.8 \mathrm{~W} \mathrm{~m}^{-1} \mathrm{~K}^{-1}$ at $300 \mathrm{~K}$ derived from low elastic properties. The promising $Z T$ value $\sim 0.2$ at $653 \mathrm{~K}$ for undoped Te motivates us to further optimize electrical transport properties via applying various dopants, such as $\mathrm{Pb}, \mathrm{Sn}$, Bi and $\mathrm{Sb}$ elements. Among these dopants, we find Bi and Sb can effectively enhance the electrical conductivity. The electrical conductivity is dramatically promoted after $\mathrm{Bi}$ and $\mathrm{Sb}$ doping, leading to a maximum power factor $\sim 16.2 \mu \mathrm{W} \mathrm{cm}{ }^{-1} \mathrm{~K}^{-2}$ in $\mathrm{Te}_{0.995} \mathrm{Bi}_{0.005}$ and $\sim 18.4 \mu \mathrm{W}$ $\mathrm{cm}^{-1} \mathrm{~K}^{-2}$ in $\mathrm{Te}_{0.985} \mathrm{Sb}_{0.015}$, respectively. Meanwhile, the lattice thermal conductivity is also largely suppressed through $\mathrm{Bi}$ and $\mathrm{Sb}$ doping. As a result, the maximum $Z T$ value can be realized $\sim 0.8$ 
in $\mathrm{Te}_{0.9925} \mathrm{Bi}_{0.0075}$ and $\sim 1.1$ in $\mathrm{Te}_{0.985} \mathrm{Sb}_{0.015}$ at $673 \mathrm{~K}$, respectively. This work uncloses the effective dopants in p-type Te system, and provides detailed information about thermoelectric performance in Te systems for researchers to further insight into enhancing thermoelectric properties.

\section{Experimental details}

\section{Raw materials}

Te block (99.999\%, Aladdin element, China), $\mathrm{Pb}$ particle (99.99\%, Aladdin element, China), Sn block (99.99\%, Aladdin element, China), Bi block (99.99\%, Aladdin element, China), Sb particle (99.99\%, Aladdin element, China).

\section{Synthesis}

Te ingots $(\sim 8 \mathrm{~g})$ were prepared by putting the high-purity materials ( $\mathrm{Te}, \mathrm{Pb}, \mathrm{Sn}, \mathrm{Bi}$, and $\mathrm{Sb}$ ) into quartz tubes, and then the tubes were evacuated to $\sim 10^{-4}$ Torr and flame-sealed. The sealed tubes were slowly heated to $873 \mathrm{~K}$ over $10 \mathrm{~h}$, kept at this temperature for $6 \mathrm{~h}$, and subsequently cooled down to room temperature. The obtained ingots were crushed into powders and densified using spark plasma sintering (SPS) in a $15 \mathrm{~mm}$ diameter graphite dies under $50 \mathrm{MPa}$ at $673 \mathrm{~K}$ for 12 minutes, disk-shaped samples with dimension of $\Phi 15 \mathrm{~mm} \times 8 \mathrm{~mm}$ were obtained.

\section{Sound velocity properties}

The longitudinal and transverse acoustic velocities were measured using an ultrasonic instrument (Ultrasonic Pulser/ Receiver Model 5058 PR, Olympus, USA). The elastic constants and Poisson ratio were calculated using the measured velocities. The uncertainty of acoustic velocity measurement is below $5 \%$.

\section{Electrical transport properties}

The SPSed samples were cut into bars with dimensions $12 \times 3$ $\times 3 \mathrm{~mm}^{3}$ for simultaneous measurement of the Seebeck coefficient and electrical conductivity using a CTA instrument under a low-pressure helium atmosphere from room temperature to $673 \mathrm{~K}$. The uncertainty of the Seebeck coefficient and electrical conductivity measurement is $5 \%$.

\section{Thermal transport properties}

The SPSed pellets were cut and polished into a shape of $\Phi 6 \mathrm{~mm}$ $\times 2 \mathrm{~mm}$ for thermal diffusivity $(D)$ measurements. The thermal diffusivity data were analyzed using a Cowan model with pulse correction. The samples were coated with a thin layer of graphite to minimize errors from the emissivity method in a Netzsch LFA457, the density $(\rho)$ was determined using the dimensions and mass of the samples, and the specific heat capacity $\left(C_{\mathrm{p}}\right)$ was derived using a reference sample (Pyroceram 9606). The thermal conductivity was calculated via $\kappa=D \rho C_{\mathrm{p}}$ and the uncertainty of the thermal conductivity is estimated to be within $8 \%$. Considering all the uncertainties from $D, \rho$ and $C_{\mathrm{p}}$, the combined uncertainty for all measurements involved in the calculation of $Z T$ is less than $20 \%$.

\section{X-ray powder diffraction}

Samples powdered with an agate mortar were used for X-ray powder diffraction. The diffraction patterns were recorded with $\mathrm{Cu} \mathrm{K}_{\alpha}(\lambda=1.5418 \AA)$ radiation in a reflection geometry on an Inel diffractometer operating at $40 \mathrm{kV}$ and $20 \mathrm{~mA}$ and equipped with a position-sensitive detector.

\section{Results and discussion}

Te samples are prepared by using melting method followed by spark plasma sintering process with high purity Te block. Because of the anisotropic crystal structure with $P 3_{1} 21$ space group ${ }^{36}$ we decide to observe the intrinsic thermoelectric properties from two different directions, namely horizontal direction perpendicular to SPS axial pressure and vertical direction parallel to SPS axial pressure. Fig. 1 shows the temperature dependent thermoelectric performance of undoped Te. The electrical conductivity is relatively low and rises slightly with increasing temperature, Fig. 1(a), which indicates a semiconductor behavior. As shown in Fig. 1(b), the Seebeck coefficients behave a similar tendency with increasing temperature in two different measured directions, however, a distinct difference can be observed especially in low temperature range. Ultimately, the calculated power factor can reach $\sim 3.4 \mu \mathrm{W} \mathrm{cm}{ }^{-1} \mathrm{~K}^{-2}$ and $\sim 2.9 \mu \mathrm{W} \mathrm{cm}{ }^{-1} \mathrm{~K}^{2}$ in horizontal direction and vertical direction respectively, as shown in Fig. 1(c). Both total thermal conductivity and lattice thermal conductivity show an obvious discrepancy between two different directions, as shown in Fig. 1(d) and (e). In Fig. 1(d), the final $Z T$ value can be achieved to $\sim 0.2$ at $653 \mathrm{~K}$ in both directions due to a low thermal conductivity, results indicate that Te exhibits an isotropic thermoelectric performance, in the following work we measured the properties along the SPS horizontal direction.

To explore the origin of the low thermal conductivity in undoped Te, the elastic measurements are carried out to measure the longitudinal and transverse acoustic velocities in Te. Based on the measured acoustic velocities, we can estimate the Young' modulus $(E)$, Grüneisen parameter $(\gamma)$ and Debye temperature $\left(\theta_{\mathrm{D}}\right)$, which are the key parameters to revealing a crystalline material's lattice thermal conductivity. ${ }^{37,38}$ As shown in Table 1, the measured longitudinal and transverse sound velocities are $2358 \mathrm{~ms}^{-1}$ and $1447 \mathrm{~ms}^{-1}$, respectively, which are consistent with reported $2285 \mathrm{~ms}^{-1}$ and $1410 \mathrm{~ms}^{-1} \cdot{ }^{36}$ As a result, we obtain relatively low average sound velocities and Young's modulus, $1597 \mathrm{~ms}^{-1}$ and $31.3 \mathrm{GPa}$ in horizontal direction, 1494 $\mathrm{ms}^{-1}$ and $27.7 \mathrm{GPa}$ in vertical direction of the sample. The Grüneisen parameter and Debye temperature are also included in Table 1. Elastic properties reveal that the weak chemical bonding strength in elementary Te system contributes largely to the low thermal conductivity.

Additionally, we provide a estimation of the lattice thermal conductivity in undoped elementary Te at room temperature using the approach suggested by Cahill: ${ }^{32,39}$ 

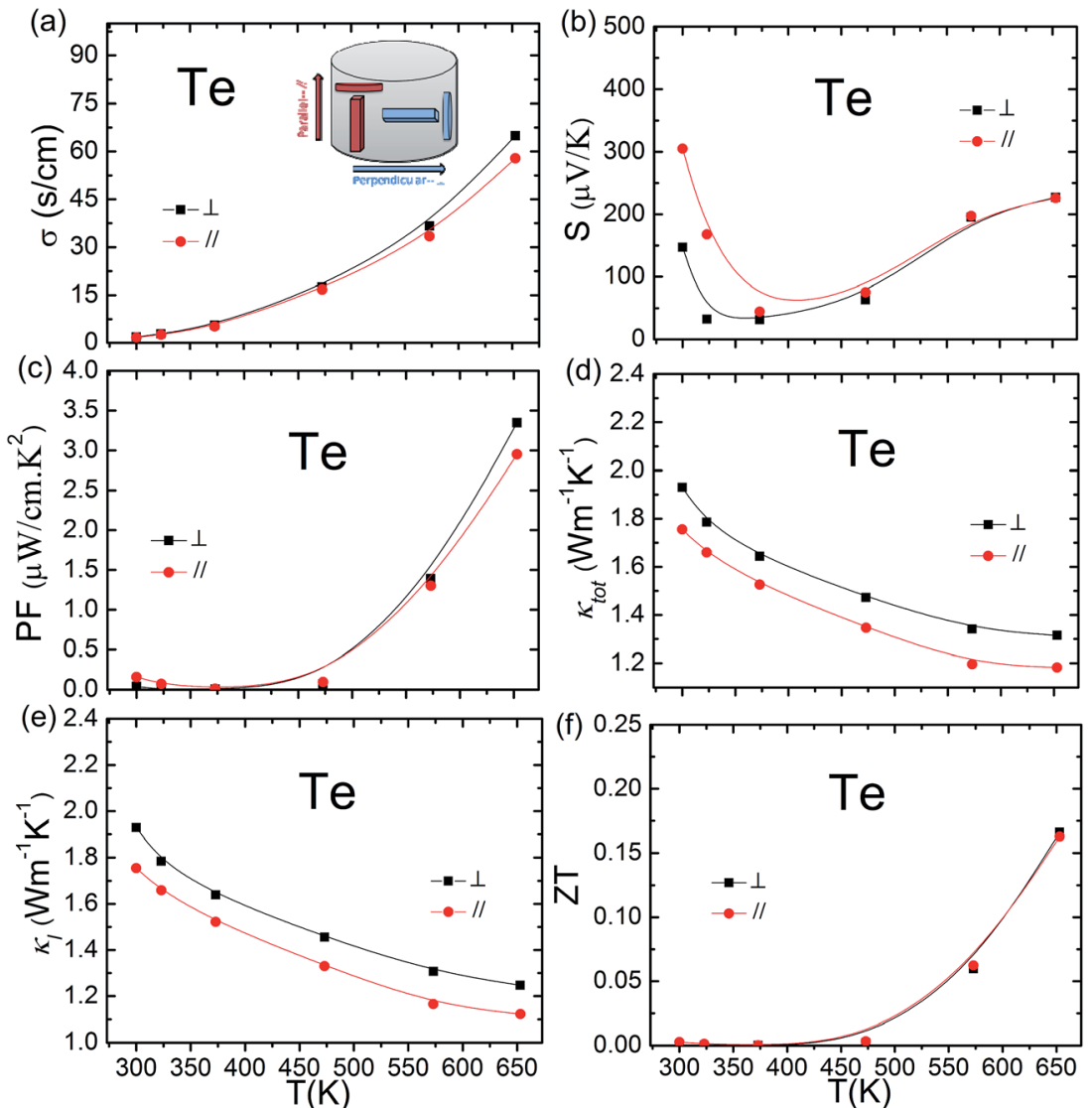

Fig. 1 Temperature dependence of thermoelectric properties in undoped Te along two directions: (a) electrical conductivity, (b) Seebeck coefficient, (c) power factor, (d) total thermal conductivity, (e) lattice thermal conductivity, and (f) ZT values.

$$
\kappa_{\min }=\left(\frac{\pi}{6}\right)^{1 / 3} k_{\mathrm{B}} n^{2 / 3} \sum_{i} v_{i}\left(\frac{T}{\theta_{i}}\right)^{2} \int_{0}^{\theta_{i} / T} \frac{x^{3} \mathrm{e}^{x}}{\left(\mathrm{e}^{x}-1\right)^{2}} \mathrm{~d} x
$$

where $v, \theta$ and $n$ denote the phonon velocity, Debye temperature and the number density of atoms, respectively. See in Table 1 , the calculated lattice thermal conductivity, $1.82 \mathrm{~W} \mathrm{~m}^{-1} \mathrm{~K}^{-1}$ and $1.7 \mathrm{~W} \mathrm{~m}^{-1} \mathrm{~K}^{-1}$ in horizontal and vertical direction, is in good accordance with the measured results.

Since undoped Te possesses a poor electrical conductivity, we carry out doping method including $\mathrm{Pb}, \mathrm{Sn}, \mathrm{Bi}$ and $\mathrm{Sb}$ elements to optimize the carrier concentration Among these

Table 1 Room temperature elastic and thermal transport properties of $\mathrm{Te}$

\begin{tabular}{lll}
\hline Parameters & $\begin{array}{l}\text { Horizontal } \\
\text { direction }\end{array}$ & $\begin{array}{l}\text { Vertical } \\
\text { direction }\end{array}$ \\
\hline Longitudinal sound velocity $\nu_{1}\left(\mathrm{~ms}^{-1}\right)$ & 2358 & 2254 \\
Transverse sound velocity $\nu_{\mathrm{s}}\left(\mathrm{ms}^{-1}\right)$ & 1447 & 1350 \\
Average sound velocity $\nu_{\mathrm{a}}\left(\mathrm{ms}^{-1}\right)$ & 1597 & 1494 \\
Young's modulus $E(\mathrm{GPa})$ & 31.3 & 27.7 \\
Grüneisen parameter $\gamma$ & 1.28 & 1.37 \\
Debye temperature $\theta_{\mathrm{D}}(\mathrm{K})$ & 147 & 137 \\
Theoretical $\kappa_{\mathrm{L}}\left(\mathrm{W} \mathrm{m} \mathrm{K}^{-1} \mathrm{~K}^{-1}\right)$ & 1.83 & 1.72 \\
Experimental $\kappa_{\mathrm{L}}\left(\mathrm{W} \mathrm{m}^{-1} \mathrm{~K}^{-1}\right)$ & 1.98 & 1.80
\end{tabular}

dopants, we find Bi and Sb can effectively enhance the electrical conductivity. Thereby, we mainly focus on Bi and Sb dopants to analyze their influence on the thermoelectric performance in $\mathrm{Te}$ system, the results of $\mathrm{Pb}$ and $\mathrm{Sn}$ doping can be found in ESI. $\dagger$ Fig. 2 shows the XRD patterns and lattice parameters varying with doping constituents in $\mathrm{Te}_{1-x} \mathrm{M}_{x}(\mathrm{M}=\mathrm{Bi}, \mathrm{Sb} ; x=0-0.02)$. As shown in Fig. 2(a) and (b), no second phase can be observed in the XRD patterns of all the doped samples and a slightly regular peak shift with increasing doping constitutes in both $\mathrm{Bi}$ and $\mathrm{Sb}$ cases. The successful incorporation of $\mathrm{Bi}$ and $\mathrm{Sb}$ atoms into the Te lattice sites can be confirmed by the increase of lattice parameters in Fig. 2(c) and (d) respectively. As shown in Fig. 3(a) and (b), the electrical conductivity obtains a remarkable increase from $2 \mathrm{~S} \mathrm{~cm}^{-1}$ in undoped Te to $\sim 319 \mathrm{~S} \mathrm{~cm}^{-1}$ and $\sim 598$ $\mathrm{S} \mathrm{cm}{ }^{-1}$ at $300 \mathrm{~K}$ in $\mathrm{Te}_{0.985} \mathrm{Bi}_{0.015}$ and $\mathrm{Te}_{0.985} \mathrm{Sb}_{0.015}$. It is noteworthy that $\mathrm{Sb}$ dopant produces a more effective role to enhance the electrical conductivity in Te. Obviously, the Seebeck coefficient gets an unexpected increase in both cases, seen in Fig. 3(c) and (d), which is attributed to the particular inherent valance band structure. ${ }^{35,36}$ When the carrier concentration increases in $\mathrm{Bi}$ and $\mathrm{Sb}$ doped samples, the Fermi level successfully crosses into more valance bands, giving rise to an enhancement of the carrier effective mass and promoting the Seebeck coefficient. Correspondingly, the power factor undergoes a dominant enhancement, Fig. 3(e) and (f). The maximum power factor is 

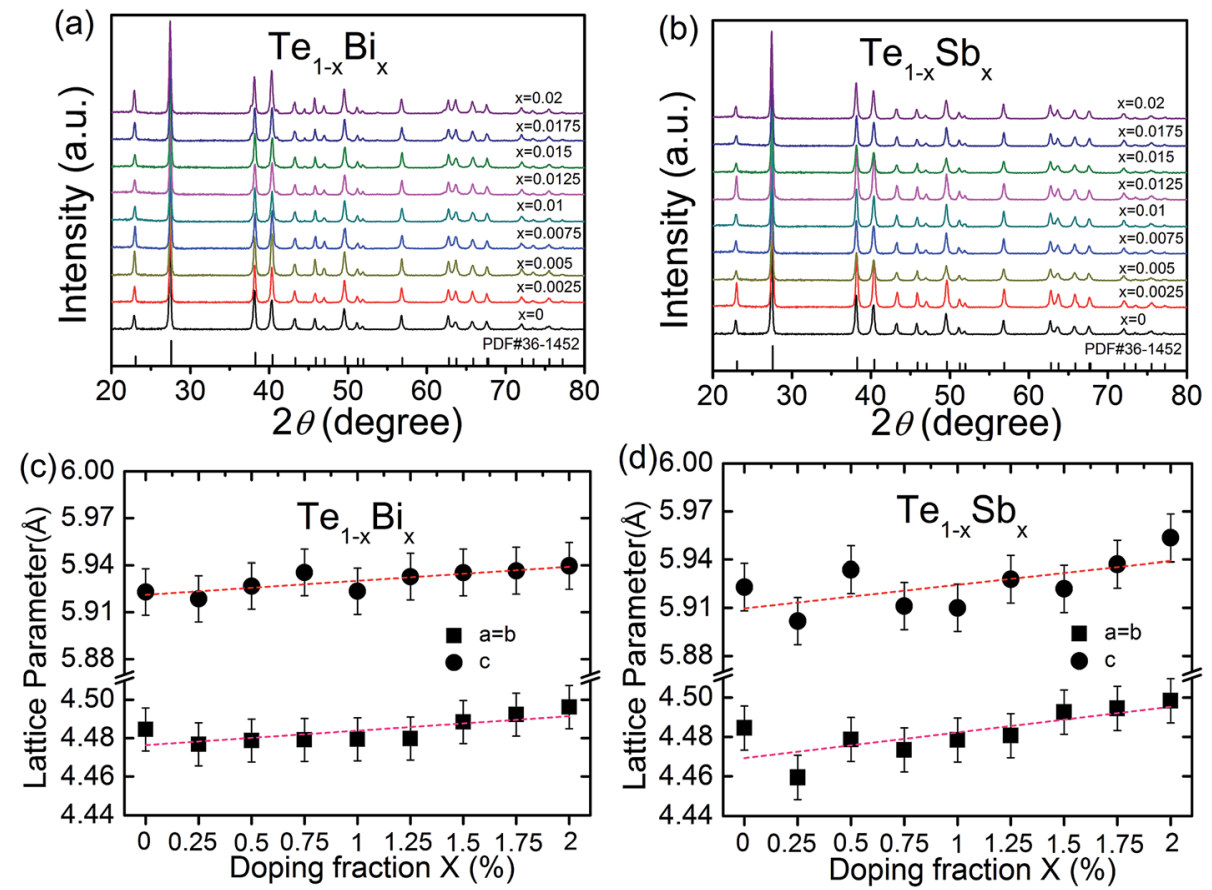

Fig. 2 (a) Powder XRD patterns and (c) lattice parameter as a function of Bi doping amount for $\mathrm{Te}_{1-x} \mathrm{Bi}_{x}(x=0-0.02)$, (b) powder XRD patterns and (d) lattice parameter as a function of $\mathrm{Sb}$ doping amount for $\mathrm{Te}_{1-x} \mathrm{Sb}_{x}(x=0-0.02)$, the red dash lines are provided to guide the eye.
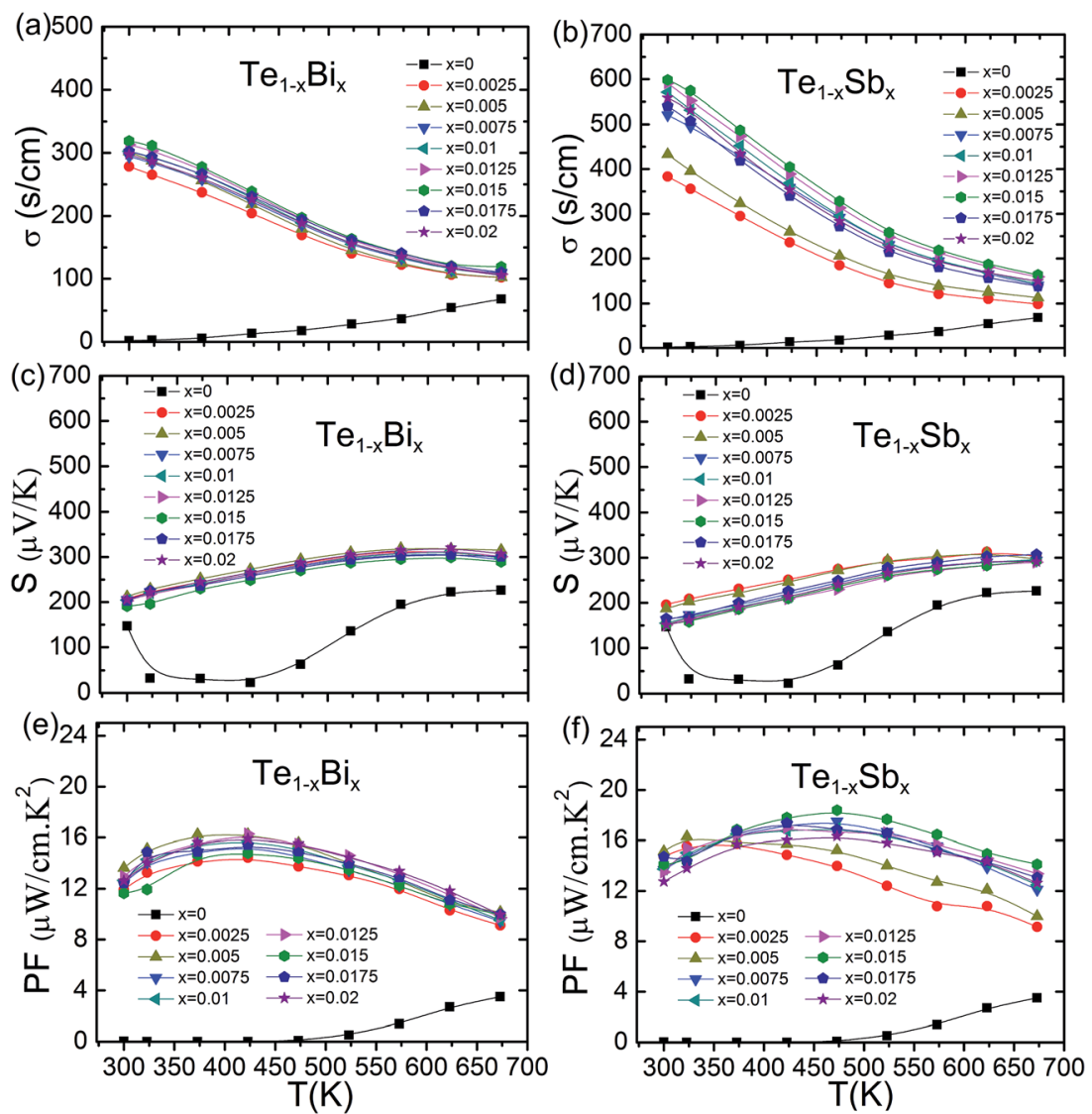

Fig. 3 Temperature dependence of electrical transport properties: (a) electrical conductivity, (c) Seebeck coefficient, (e) power factor in Te $e_{1-x} \mathrm{Bi}_{x}$ $(x=0-0.02)$, (b) electrical conductivity, (d) Seebeck coefficient, (f) power factor in $\mathrm{Te}_{1-x} \mathrm{Sb}_{x}(x=0-0.02)$. 

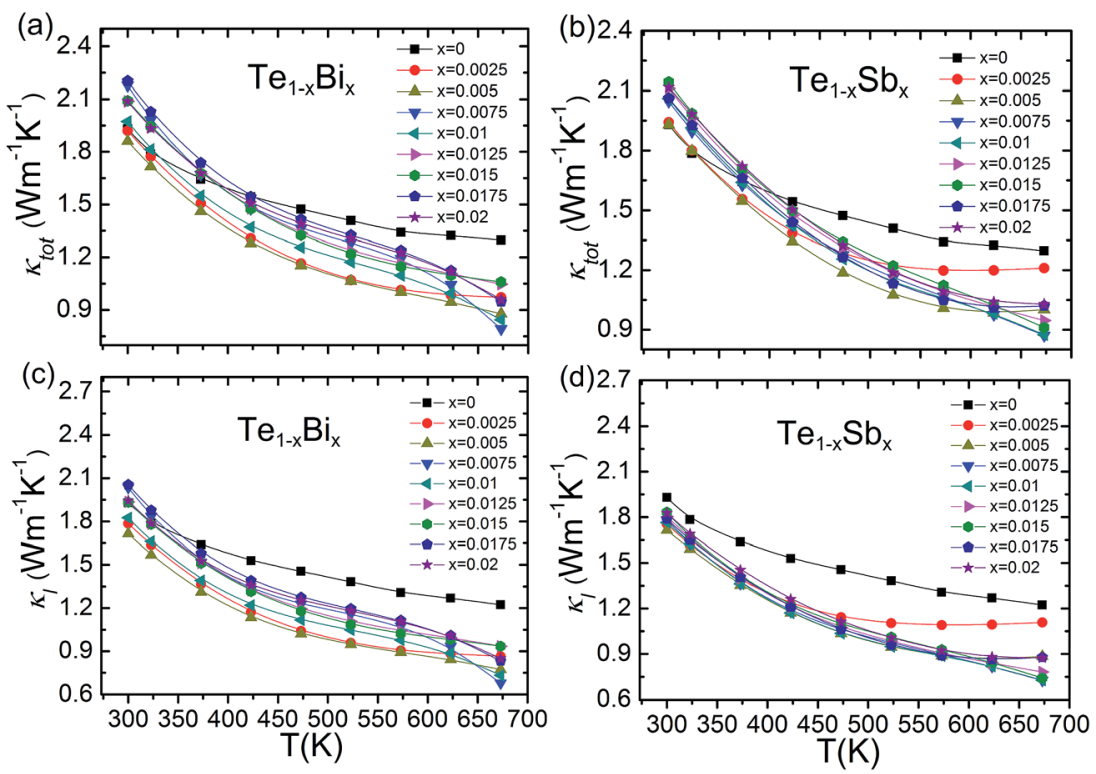

Fig. 4 Temperature dependence of thermal transport properties: (a) total thermal conductivity, (c) lattice thermal conductivity in Te $e_{1-x} \mathrm{Bi}_{x}(x=$ $0-0.02)$, (b) total thermal conductivity, (d) lattice thermal conductivity in $\mathrm{Te}_{1-x} \mathrm{Sb}_{x}(x=0-0.02)$.
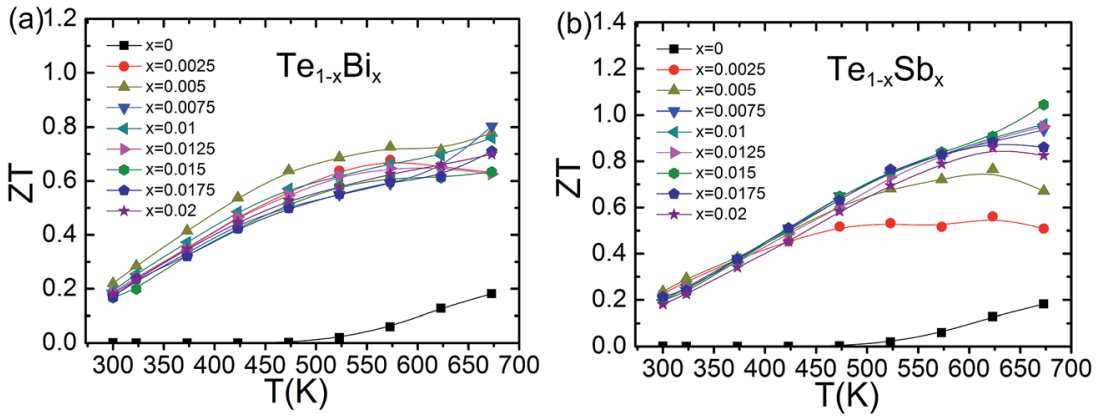

Fig. 5 Temperature dependence of $Z T$ values: (a) $\mathrm{Te}_{1-x} \mathrm{Bi}_{x}(x=0-0.02)$, (b) $\mathrm{Te}_{1-x} \mathrm{Bi}_{x}(x=0-0.02)$.

amplified from $\sim 3.5 \mu \mathrm{W} \mathrm{cm}{ }^{-1} \mathrm{~K}^{-2}$ in undoped Te sample to $\sim 16.2 \mu \mathrm{W} \mathrm{cm}{ }^{-1} \mathrm{~K}^{-2}$ in $\mathrm{Te}_{0.995} \mathrm{Bi}_{0.005}$ and $\sim 18.4 \mu \mathrm{W} \mathrm{cm}{ }^{-1} \mathrm{~K}^{-2}$ in $\mathrm{Te}_{0.985} \mathrm{Sb}_{0.015}$, respectively. Noticeably, the power factor in Sbdoped samples possesses higher values than that of Bi-doped samples owing to the superior electrical conductivity.

After Bi and Sb doping, the total thermal conductivity decreases largely, Fig. 4(a) and (b), which is mainly derived from the reduction of lattice thermal conductivity especially in elevated temperature range, as shown in Fig. 4(c) and (d). The lattice thermal conductivity part is significantly suppressed from $1.22 \mathrm{~W} \mathrm{~m}^{-1} \mathrm{~K}^{-1}$ in undoped Te to $0.68 \mathrm{~W} \mathrm{~m}^{-1} \mathrm{~K}^{-1}$ and $0.73 \mathrm{~W} \mathrm{~m}^{-1} \mathrm{~K}^{-1}$ at $673 \mathrm{~K}$ in $\mathrm{Te}_{0.9925} \mathrm{Bi}_{0.0075}$ and $\mathrm{Te}_{0.985} \mathrm{Sb}_{0.015}$, respectively. This phenomenon indicates that the point defects derived from doping can intensify phonon scattering and make the phonon propagation slowly. ${ }^{39,40}$ In combination with the largely improved power factor, the maximum $Z T$ values can achieve $\sim 0.8$ in $\mathrm{Te}_{0.9925} \mathrm{Bi}_{0.0075}$ and $\sim 1.1$ in $\mathrm{Te}_{0.985} \mathrm{Sb}_{0.015}$ at $673 \mathrm{~K}$ respectively, Fig. 5 .

Compared with the previously report, Fig. 6(a), both the samples with $n=2.65 \times 10^{19} \mathrm{~cm}^{-3}$ and $n=3 \times 10^{19} \mathrm{~cm}^{-3}$ (ref. 36) exhibit higher electrical conductivity than that of $\mathrm{Bi}$ and $\mathrm{Sb}$ doped samples. The Seebeck coefficient decreases with increasing carrier concentration, as shown in Fig. 6(b), which leads to a declining tendency in power factor, Fig. $6(\mathrm{c})$. The maximum value of power factor in the sample with $n=2.65 \times 10^{19} \mathrm{~cm}^{-3}$ reaches $\sim 17.6 \mu \mathrm{W}$ $\mathrm{cm}^{-1} \mathrm{~K}^{-2}$ at $550 \mathrm{~K}$, which is comparable to the present $\mathrm{Te}_{0.985} \mathrm{Sb}_{0.015}$ sample. The sample with $n=2.65 \times 10^{19} \mathrm{~cm}^{-3}$ (ref. 36) also possess a similar total thermal conductivity with $\mathrm{Te}_{0.985} \mathrm{Sb}_{0.015}$ sample, Fig. 6(d), even though they own much higher electrical conductivity related to higher electronic thermal conductivity. The relatively low total thermal conductivity primarily comes from the dramatically reduced lattice thermal conductivity, as shown in Fig. 6(e). As a consequence, the present results show that the $\mathrm{Te}_{0.985} \mathrm{Sb}_{0.015}$ sample in this work can exhibit $Z T$ value of 1.0 at $673 \mathrm{~K}$, which is comparable to the reported value in the sample with carrier concentration of $n=2.65 \times 10^{19} \mathrm{~cm}^{-3} \cdot{ }^{36}$

\section{Conclusions}

This work investigates the thermoelectric performance of undoped Te and illustrates its low thermal conductivity using sound velocity measurements. Importantly, we unclose the detailed effects of $\mathrm{Bi}$ and $\mathrm{Sb}$ dopants on the thermoelectric 

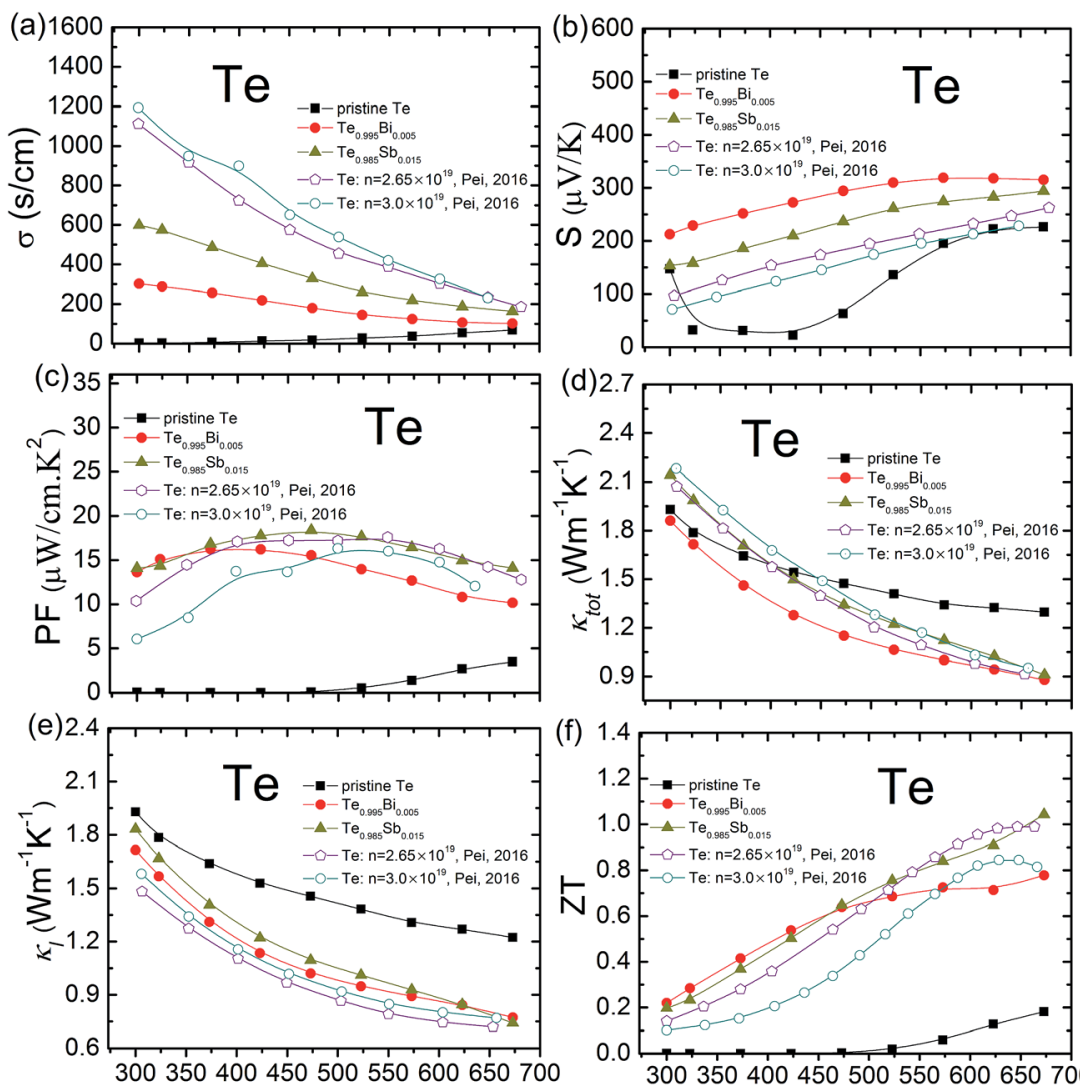

$\mathrm{T}(\mathrm{K})$

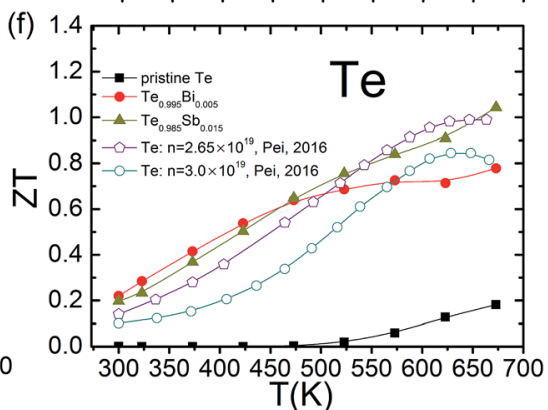

Fig. 6 Temperature dependence of thermoelectric properties of the samples in this work and its comparisons: (a) electrical conductivity, (b) Seebeck coefficient, (c) power factor, (d) total thermal conductivity, (e) lattice thermal conductivity, (f) $Z T$ values.

performance of elementary $\mathrm{Te}$. Bi and Sb dopants can not only enhance the power factor also largely suppress the lattice thermal conductivity, which clarifies the effective dopants in Te that can guideline researchers to further focus on enhancing thermoelectric performance in Te system. Indeed, a more excellent thermoelectric performance deserves to be expected in Te system if carrier concentration is properly optimized in combination with further reduced thermal conductivity using nanostructuring.

\section{Acknowledgements}

This work was supported by NSFC under Grant No. 51571007 . This work was also supported by Shenzhen peacock plan team Grant No. KQTD2016022619565911.

\section{References}

1 G. Tan, L.-D. Zhao and M. G. Kanatzidis, Rationally Designing High-Performance Bulk Thermoelectric Materials, Chem. Rev., 2016, 116(19), 12123-12149.

2 X. Zhang and L.-D. Zhao, Thermoelectric materials: energy conversion between heat and electricity, Journal of Materiomics, 2015, 1(2), 92-105.

3 L.-D. Zhao, V. P. Dravid and M. G. Kanatzidis, The panoscopic approach to high performance thermoelectrics, Energy Environ. Sci., 2014, 7(1), 251-268.
4 G. J. Tan, et al., Codoping in SnTe: Enhancement of Thermoelectric Performance through Synergy of Resonance Levels and Band Convergence, J. Am. Chem. Soc., 2015, 137(15), 5100-5112.

5 G. J. Tan, et al., Valence Band Modification and High Thermoelectric Performance in SnTe Heavily Alloyed with MnTe, J. Am. Chem. Soc., 2015, 137(35), 11507-11516.

6 L. D. Zhao, et al., All-scale hierarchical thermoelectrics: MgTe in PbTe facilitates valence band convergence and suppresses bipolar thermal transport for high performance, Energy Environ. Sci., 2013, 6(11), 3346.

$7 \mathrm{H}$. Wu, et al., Strong enhancement of phonon scattering through nanoscale grains in lead sulfide thermoelectrics, NPG Asia Mater., 2014, 6(6), e108.

8 W. S. Liu, et al., Effects of Sb compensation on microstructure, thermoelectric properties and point defect of $\mathrm{CoSb}_{3}$ compound, J. Phys. D: Appl. Phys., 2007, 40(21), 6784-6790.

9 K. Biswas, L.-D. Zhao and M. G. Kanatzidis, Tellurium-Free Thermoelectric: The Anisotropic n-Type Semiconductor $\mathrm{Bi}_{2} \mathrm{~S}_{3}$, Adv. Energy Mater., 2012, 2(6), 634-638.

$10 \mathrm{G}$. Tan, et al., Extraordinary role of $\mathrm{Hg}$ in enhancing the thermoelectric performance of p-type SnTe, Energy Environ. Sci., 2015, 8(1), 267-277.

11 L.-D. Zhao, et al., Effect of mixed grain sizes on thermoelectric performance of $\mathrm{Bi}_{2} \mathrm{Te}_{3}$ compound, J. Appl. Phys., 2009, 105(2), 023704. 
12 L. D. Zhao, et al., Thermoelectrics with earth abundant elements: high performance p-type $\mathrm{PbS}$ nanostructured with SrS and CaS, J. Am. Chem. Soc., 2012, 134(18), 79027912.

13 Y.-L. Pei, et al., High thermoelectric performance of oxyselenides: intrinsically low thermal conductivity of Cadoped BiCuSeO, NPG Asia Mater., 2013, 5(5), e47.

$14 \mathrm{~J}$. Sui, et al., Texturation boosts the thermoelectric performance of BiCuSeO oxyselenides, Energy Environ. Sci., 2013, 6(10), 2916.

$15 \mathrm{~F}$. Li, et al., Polycrystalline BiCuSeO oxide as a potential thermoelectric material, Energy Environ. Sci., 2012, 5(5), 7188.

16 L. D. Zhao, et al., Enhanced Thermoelectric Properties in the Counter-Doped SnTe System with Strained Endotaxial SrTe, J. Am. Chem. Soc., 2016, 138(7), 2366-2373.

17 W. S. Liu, et al., Thermoelectric Property Studies on CuDoped n-type $\mathrm{Cu}_{x} \mathrm{Bi}_{2} \mathrm{Te}_{2.7} \mathrm{Se}_{0.3}$ Nanocomposites, Adv. Energy Mater., 2011, 1(4), 577-587.

18 J. S. Son, et al., n-Type Nanostructured Thermoelectric Materials Prepared from Chemically Synthesized Ultrathin $\mathrm{Bi}_{2} \mathrm{Te}_{3}$ Nanoplates, Nano Lett., 2012, 12(2), 640-647.

19 Z. J. Xu, et al., Attaining high mid-temperature performance in $(\mathrm{Bi}, \mathrm{Sb})_{2} \mathrm{Te}_{3}$ thermoelectric materials via synergistic optimization, NPG Asia Mater., 2016, 8, e302.

20 S. Johnsen, et al., Nanostructures boost the thermoelectric performance of PbS, J. Am. Chem. Soc., 2011, 133(10), 3460-3470.

$21 \mathrm{~J}$. P. Heremans, et al., Enhancement of thermoelectric efficiency in PbTe by distortion of the electronic density of states, Science, 2008, 321(5888), 554-557.

22 M. Zhou, J.-F. Li and T. Kita, Nanostructured AgPb ${ }_{m} \mathrm{SbTe}_{m+2}$ System Bulk Materials with Enhanced Thermoelectric Performance, J. Am. Chem. Soc., 2008, 130(13), 4527-4532.

23 E. S. Toberer, A. F. May and G. J. Snyder, Zintl Chemistry for Designing High Efficiency Thermoelectric Materials, Chem. Mater., 2010, 22(3), 624-634.

$24 \mathrm{X}$. Shi, et al., Multiple-Filled Skutterudites: High Thermoelectric Figure of Merit through Separately Optimizing Electrical and Thermal Transports, J. Am. Chem. Soc., 2011, 133(20), 7837-7846.

25 G. Rogl, et al., Thermoelectric properties of novel skutterudites with didymium: $\mathrm{DDy}\left(\mathrm{Fe}_{1-x} \mathrm{Co}_{x}\right)_{4} \mathrm{Sb}_{12}$ and $\operatorname{DDy}\left(\mathrm{Fe}_{1-x} \mathrm{Ni}_{x}\right)_{4} \mathrm{Sb}_{12}$, Intermetallics, 2010, 18(1), 57-64.
26 W. Y. Zhao, et al., Enhanced Thermoelectric Performance in Barium and Indium Double-Filled Skutterudite Bulk Materials via Orbital Hybridization Induced by Indium Filler, J. Am. Chem. Soc., 2009, 131(10), 3713-3720.

27 H. L. Liu, et al., Copper ion liquid-like thermoelectrics, Nat. Mater., 2012, 11(5), 422-425.

28 D. W. Yang, et al., Mechanochemical synthesis of high thermoelectric performance bulk $\mathrm{Cu}_{2} \mathrm{X}(\mathrm{X}=\mathrm{S}$, Se $)$ materials, APL Mater., 2016, 4(11), 116110.

29 G. Tan, et al., Codoping in SnTe: Enhancement of Thermoelectric Performance through Synergy of Resonance Levels and Band Convergence, J. Am. Chem. Soc., 2015, 137(15), 5100-5112.

30 L. D. Zhao, et al., $\mathrm{Bi}_{1-x} \mathrm{Sr}_{x} \mathrm{CuSeO}$ oxyselenides as promising thermoelectric materials, Appl. Phys. Lett., 2010, 97(9), 092118.

31 J. L. Lan, et al., Enhanced thermoelectric properties of $\mathrm{Pb}$ doped BiCuSeO ceramics, Adv. Mater., 2013, 25(36), 50865090.

32 L. D. Zhao, et al., Ultralow thermal conductivity and high thermoelectric figure of merit in SnSe crystals, Nature, 2014, 508(7496), 373-377.

33 L. D. Zhao, et al., Ultrahigh power factor and thermoelectric performance in hole-doped single-crystal SnSe, Science, 2016, 351(6269), 141-144.

34 L. A. Agapito, et al., Novel family of chiral-based topological insulators: elemental tellurium under strain, Phys. Rev. Lett., 2013, 110(17), 2368-2373.

35 H. Peng, N. Kioussis and G. J. Snyder, Elemental tellurium as a chiral p-type thermoelectric material, Phys. Rev. B: Condens. Matter Mater. Phys., 2014, 89(19), 195206.

36 S. Q. Lin, et al., Tellurium as a high-performance elemental thermoelectric, Nat. Commun., 2016, 7, 10287.

$37 \mathrm{Y}$. Xiao, et al., Electrical and thermal transport properties of layered $\mathrm{Bi}_{2} \mathrm{YO}_{4} \mathrm{Cu}_{2} \mathrm{Se}_{2}$, J. Solid State Chem., 2016, 239, 178183.

38 Y. Xiao, et al., Origin of low thermal conductivity in SnSe, Phys. Rev. B, 2016, 94(12), 125203.

39 D. G. Cahill, S. K. Watson and R. O. Pohl, Lower limit to the thermal conductivity of disordered crystals, Phys. Rev. B: Condens. Matter Mater. Phys., 1992, 46(10), 6131-6140.

$40 \mathrm{~J}$. Callaway and H. C. von Baeyer, Effect of point imperfections on lattice thermal conductivity, Phys. Rev., 1960, 120(4), 1149-1154. 